\title{
A ptosis repair of aponeurotic defects by the posterior approach
}

\author{
J. R. O. COLLIN
}

From the Department of Clinical Ophthalmology, Moorfields Eye Hospital, and the Institute of Ophthalmology, London

SUMMARY A simple posterior approach operation for correcting ptosis due to a disinsertion of the aponeurosis of the levator palpebrae superioris muscle is presented. Müller's muscle is restored to its normal length. Pull-out sutures are used which give some postoperative control of eyelid level and allow the procedure to be done under general anaesthesia if required.

Patients with an aponeurotic defect have a ptosis with good levator function, a thinned eyelid, and a high-arched lid fold. The affected eyelid remains lower than the other eyelid in all positions of gaze. Jones et al. (1975) first demonstrated the various aponeurotic defects which may occur in these patients (Figs. 1 and 2) and described their repair via an anterior skin approach under local anaesthesia. Although excellent results can be obtained by their technique, the results are not always

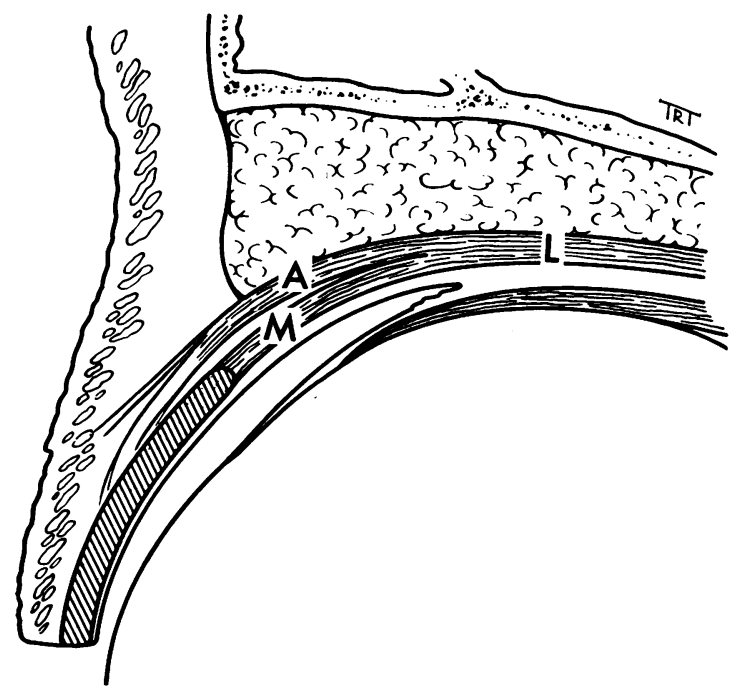

Fig. 1 Normal upper eyelid anatomy. $\mathrm{L}=$ Levator palpebrae superioris muscle. $\mathrm{M}=$ Müller's muscle. $\mathrm{A}=$ Aponeurosis

Correspondence to Mr J. R. O. Collin, FRCS, Moorfields Eye Hospital, City Road, London ECIV 2PD.

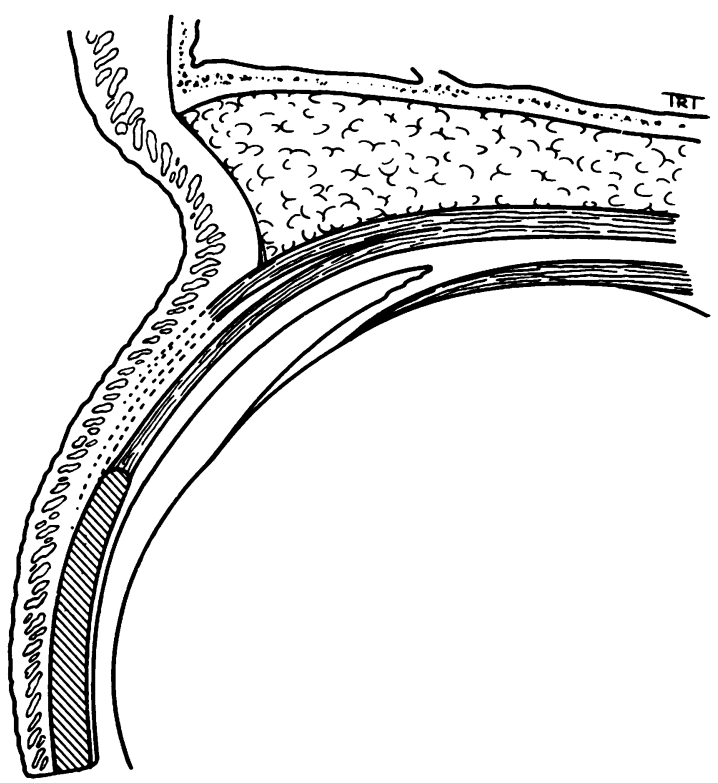

Fig. 2 Diagram of disinsertion of the aponeurosis

predictable, and the eyelid level cannot easily be adjusted postoperatively. This paper describes a posterior approach operation for the repair of aponeurotic defects which can be done under local or general anaesthesia. Pull-out sutures are used, and the eyelid level can be adjusted postoperatively.

\section{Procedure}

The operation can be done under local or general anaesthesia. Local anaesthesia is preferred, as it is easier to identify the aponeurosis with the patient's co-operation. The eyelid is infiltrated subconjunc- 


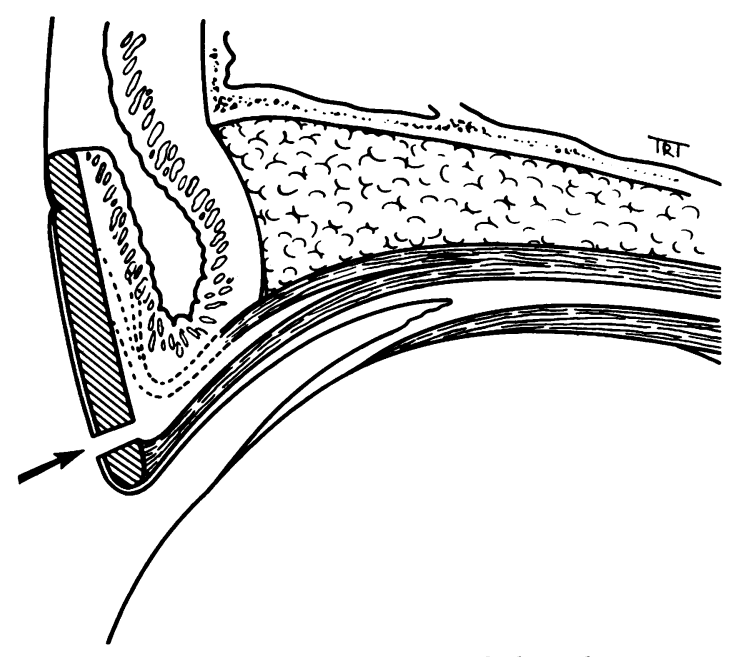

Fig. 3 Incision through everted tarsal plate about 1-2 $\mathrm{mm}$ from its upper border

tivally and subcutaneously with $2 \%$ lignocaine and $1 / 100000$ adrenaline. A 4 ' 0 ' black silk suture is placed in the tarsal plate and the eyelid everted over a Desmarres retractor. An incision is made through the tarsal plate about $1-2 \mathrm{~mm}$ from its upper border (Fig. 3). The conjunctiva is then separated from the lower part of Müller's muscle (Fig. 4). This plane is easily identified if the local anaesthetic has been injected here at the start of the operation. The conjunctiva is preserved, but the lower $2 \mathrm{~mm}$ of Müller's muscle with its tendon of insertion and the upper 1-2 mm of the tarsal plate are excised.

The aponeurosis is identified by its movement when the patient looks up and down if the operation is being done under local anaesthesia. Under general anaesthesia the orbital septum is identified first by its attachment to the orbital rim. The septum is then incised and the preaponeurotic fat pad is gently retracted. When the orbital septum has been divided, the superior surface of the aponeurosis can be examined to ensure that there is no defect posterior to the septal attachment. Both needles of 3 double-armed 5 ' 0 ' silk sutures are then passed through the conjunctiva about 2-3 mm from its cut edge, through the lower end of the shortened Müller's muscle, and through the lowest part of the aponeurosis which looks healthy. The sutures are then passed down the anterior surface of the tarsal plate and are brought out through the pretarsal orbicularis and skin at, or just below, the required position of the skin crease (Fig. 5). They are then tied over rubber or cotton-wool bolsters. This closes the conjunctiva, brings the lower cut end of Müller's muscle against the upper cut edge of the

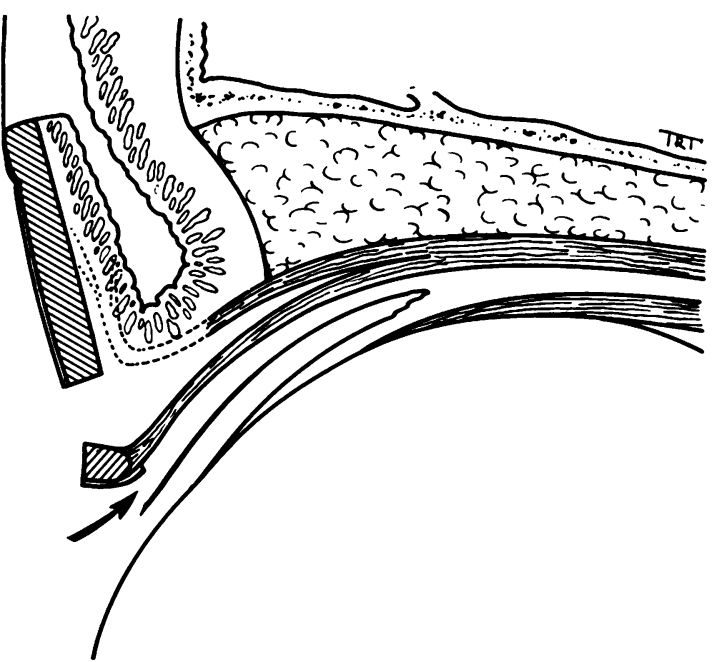

Fig. 4 Separation of conjunctiva from Müller's muscle

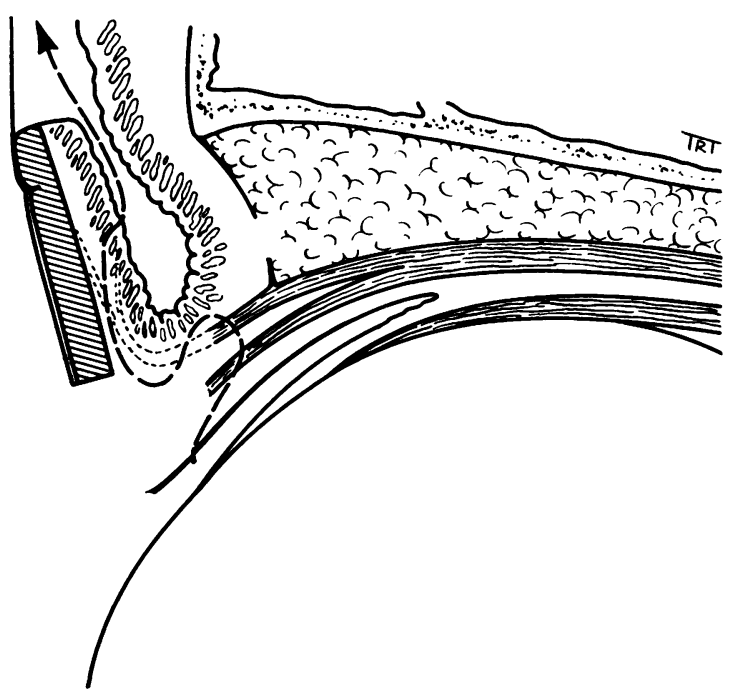

Fig. 5 Position of double-armed posteriorly placed pull-out sutures

tarsal plate, and pulls healthy aponeurotic tissue into the pretarsal orbicularis muscle to reform the skin crease (Fig. 6).

The central suture is positioned first and tightened with a temporary knot. Under local anaesthesia this will give some idea of the elevation of the eyelid. If it is too low, the suture can be repositioned to pick up the aponeurosis more proximally. If the eyelid level is too high the aponeurosis may be picked up more distally or the suture tied less tightly if there is no distal aponeurotic tissue which 


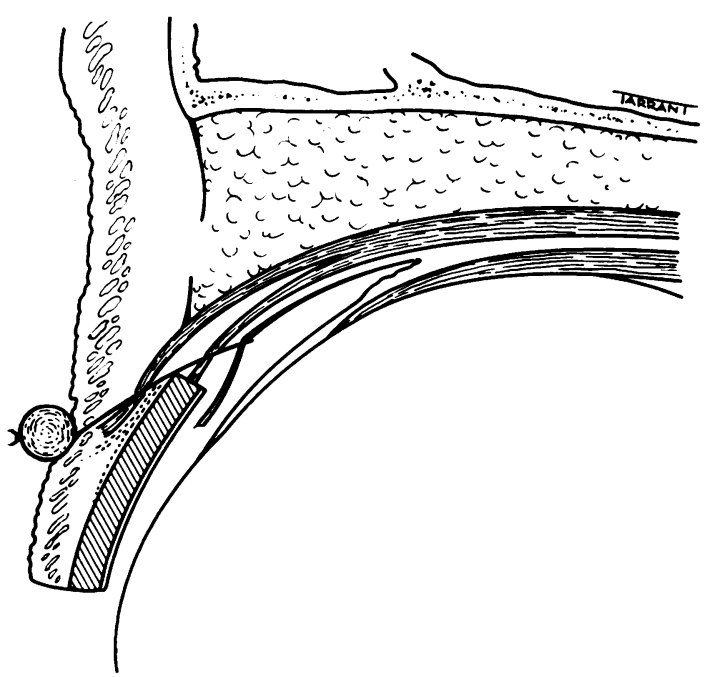

Fig. 6 Eyelid structures reapposed after tying sutures over bolsters
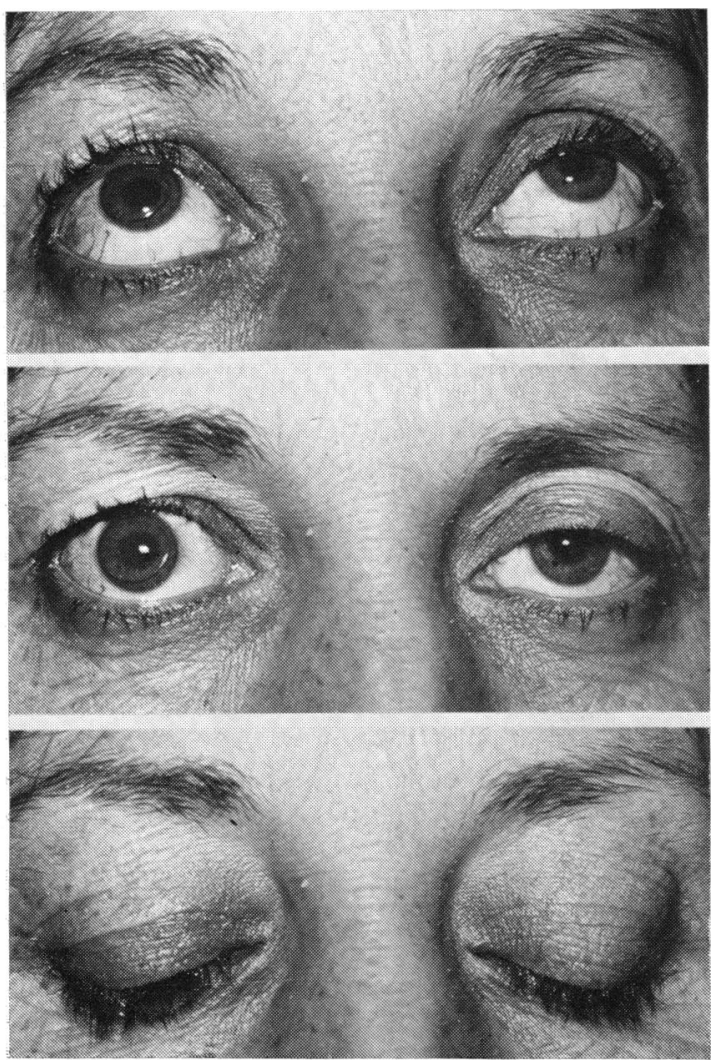

Fig. 7 Preoperatively showing a left ptosis with the clinical feature of a disinsertion of the aponeurosis appears healthy. The contour of the eyelid is then adjusted by the positioning of the medial and lateral sutures. No Frost suture is required, but an antibiotic ointment is liberally applied.

The time of suture removal is governed by the height of the eyelid postoperatively. The eyelid can be lowered by early suture removal and if need be the posterior wound can be opened with an iris repositor to let the eyelid down further. Usually the

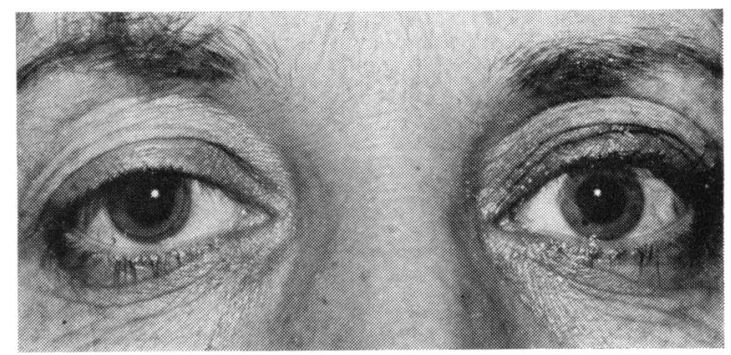

Fig. 8 Nineteen days postoperatively showing overcorrection of the left upper eyelid
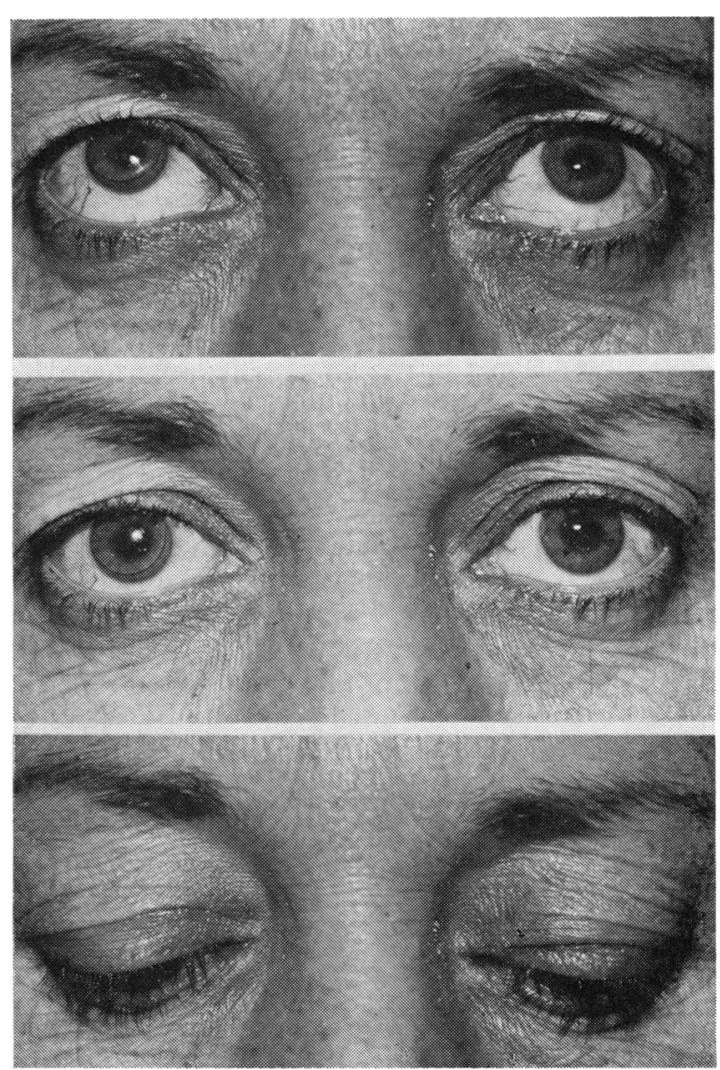

Fig. 9 Four months postoperatively showing final results after massage 

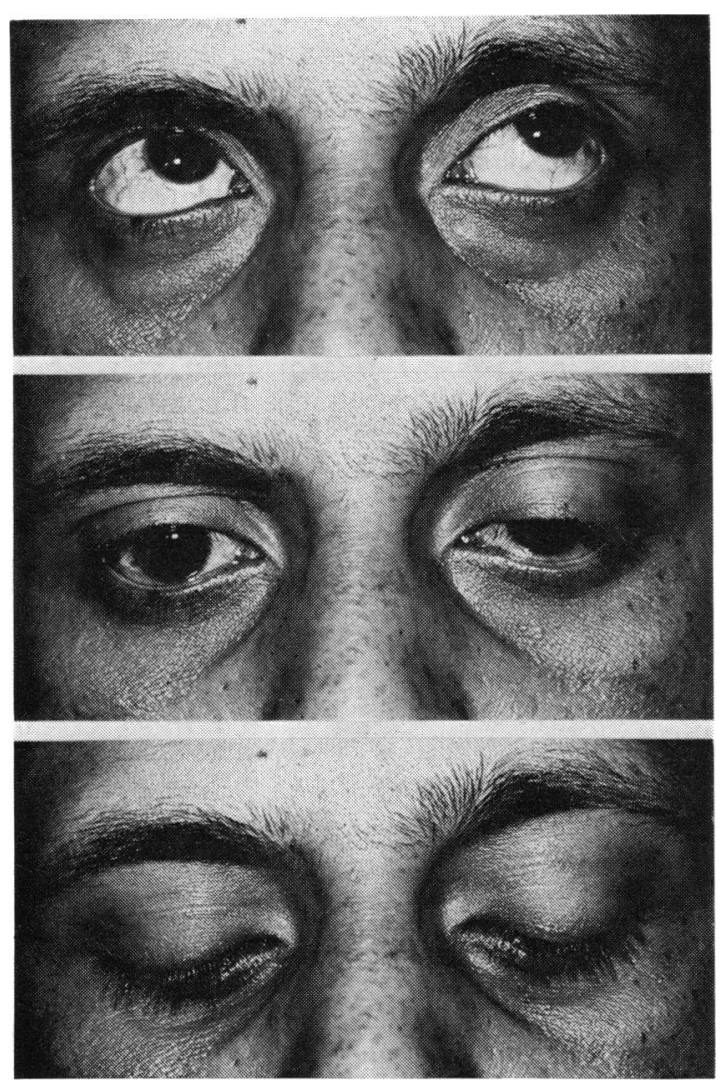

Fig. 10 Preoperatively showing a left ptosis with the clinical feature of a disinsertion of the aponeurosis

eyelid is low postoperatively, and as the oedema settles the eyelid level rises. The sutures are left for 10 days unless the eyelid level is higher than the normal side before then. If this occurs, the sutures are removed at that time. After suture removal the eyelid level is closely watched. If it becomes too high (Figs. 7 and 8), massage will bring the eyelid level down and maintain it at the correct level (Fig. 9). If it is too low, the eyelid should be left severely alone until the postoperative fibrosis raises the eyelid to the required height (Figs. 10 and 11).

\section{Results}

Three patients have been operated on with this technique. All had $2-3 \mathrm{~mm}$ of an acquired ptosis with good levator function (10-14 mm), a higharched lid fold, and a thin eyelid (Figs. 7 and 10). In the immediate postoperative period all the operated eyelids were low. The sutures were removed
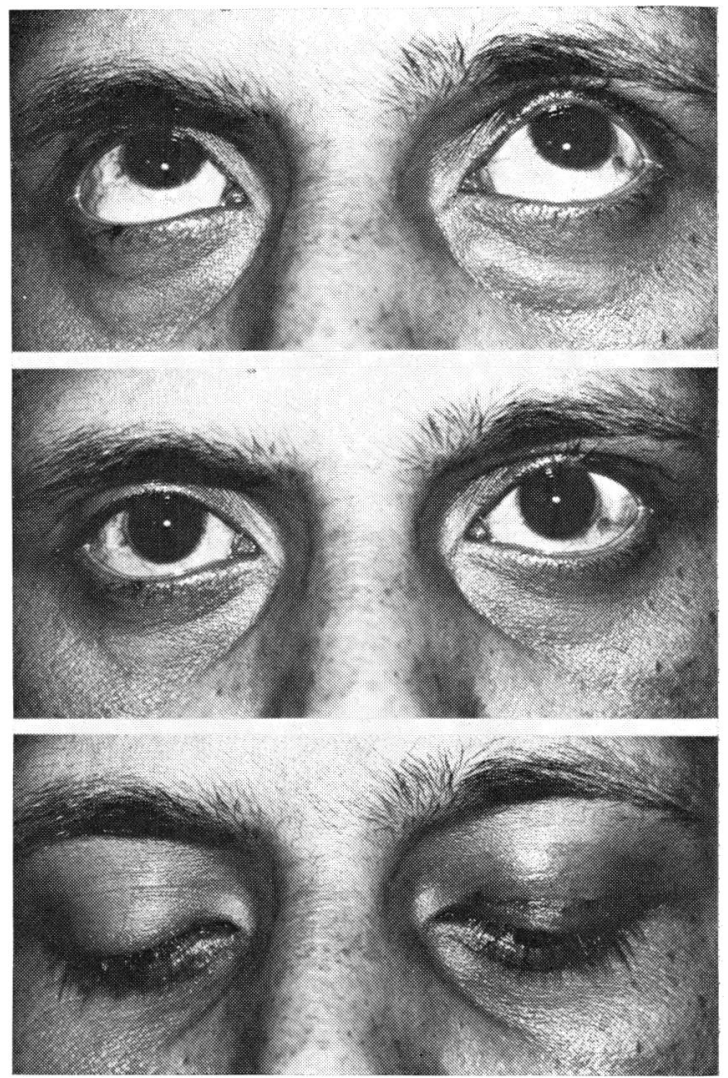

Fig. 11 Four months postoperatively showing final result without massage

after 10 days, and the eyelid level continued to rise. In 2 cases, the eyelid reached and maintained the same level as the unoperated side (Fig. 11). In the other case the operated eyelid rose too high (Fig. 8), but the appropriate level was achieved and maintained by massage (Fig. 9). All have retained a satisfactory eyelid level, contour, and skin crease for 6 months postoperatively. Three more eyelids have since been operated on with initially good results, but the follow-up period is as yet too short for comment.

\section{Discussion}

The aim of this procedure is to correct the ptosis by reinserting the aponeurosis and to restore Müller's muscle to its normal length. The eyelid level will rise in patients with an aponeurotic defect if Müller's muscle is stimulated with cocaine (Jones et al., 1975). This shows that Müller's muscle is not denervated. Histology and electron microscopy have 
not shown any defect of Müller's muscle cells (Kuwabara et al., 1975) and there is no evidence of a disinsertion of the muscle (Collin et al., 1978). Since the eyelid is ptotic, Müller's muscle must have stretched under the weight of the eyelid. It is logical to correct the effect of this stretching by shortening the muscle. If the aponeurotic defect is repaired by a standard anterior approach, Müller's muscle is not shortened as it is in the procedure reported here. Another benefit of this procedure is the use of pullout sutures, which allow a measure of postoperative control of the eyelid level. This permits the operation to be done under general anaesthesia if required with a greater degree of accuracy than with the standard anterior approach.

The disadvantages of the posterior approach over the standard anterior approach are that an incision is made through the conjunctiva and some conjunctiva and tarsal plate are sacrificed even if the amount is minimal. A small loop of each pull-out suture does pass through the conjunctiva and could irritate the cornea, but this has not happened with any cases, probably because the sutures are above the upper border of the tarsal plate and are protected by the postoperative oedema of the conjunctiva. With the anterior approach it is easier to excise any excess skin and to examine the aponeurosis for any possible defect posterior to the orbital septum. Nonabsorbable sutures can be used with an anterior approach to suture the aponeurosis to the anterior tarsus, and this may reduce the chance of a recurrent disinsertion. The follow-up period with this series is too short to comment on the chance of recurrence after this operation.

This procedure is a modification of the ptosis operation described by Werb (1976). In his operation the ptosis is corrected by resecting a large part, or all, of Müller's muscle, and if this proves insufficient he then proceeds to the resection of part of the levator muscle. This is an excellent procedure for correcting many kinds of ptosis, but in patients with an aponeurotic defect it is not necessary to excise Müller's muscle. The operation described here restores the normal length of Müller's muscle and corrects the ptosis by reinserting the aponeurosis. This re-establishes the normal eyelid anatomy as much as possible (Collin et al., 1978).

\section{References}

Collin, J. R. O., Beard, C., and Wood, I. (1978). Experimental and clinical data on the insertion of the levator palpebrae superioris muscle. American Journal of Ophthalmology, 85, 792-801.

Jones, L. T., Quickert, M. H., and Wobig, J. L. (1975). The cure of ptosis by aponeurotic repair. Archives of Ophthalmology, 93, 629-634.

Kuwabara, T., Cogan, D. G., and Johnson, C. C. (1975). Structure of the muscles of the upper eyelid. Archives of Ophthalmology, 93, 1189-1197.

Werb, A. (1976). Ptosis. Australian Journal of Ophthalmology, 4, 40-43. 